\title{
Lattice and Schröder paths with periodic boundaries
}

\author{
Joseph P.S. Kung ${ }^{1}$, Anna de Mier ${ }^{2,5}$, Xinyu Sun $^{3}$, and Catherine Yan ${ }^{4,6}$ \\ ${ }^{1}$ Department of Mathematics, \\ University of North Texas, Denton, TX 76203, U.S.A. and \\ Centre de Recerca Matemàtica, 08193 Bellaterra, Spain \\ ${ }^{2}$ Department of Applied Mathematics 2, \\ Universitat Politècnica de Catalunya, Jordi Girona 1-3, 08034 Barcelona, Spain \\ ${ }^{3}$ Mathematics Department, \\ Tulane University, 6823 St. Charles Avenue, New Orleans, LA 70118, U.S.A. \\ ${ }^{4}$ Department of Mathematics \\ Texas A\&M University, College Station, TX 77843, U.S.A. and \\ Center for Combinatorics, LPMC-TJKLC, \\ Nankai University, Tianjin 300071, P.R. China \\ ${ }^{1}$ kung@unt.edu, ${ }^{2}$ anna.de.mier@upc.edu, ${ }^{3}$ xsun1@tulane.edu, ${ }^{4}$ cyan@math.tamu.edu
}

November 1, 2018

Key words and phrases. Lattice path, Schröder path, parking function, Appell relation, algebraic generating function, the tennis ball problem

Mathematics Subject Classification. Primary 05A15; Secondary 05A10 05A40

\footnotetext{
${ }^{5}$ The second author was supported by the "Ramón y Cajal" programme of the Spanish Ministry of Science and Technology.

${ }^{6}$ The fourth author was supported in part by NSF grants DMS-0245526 and DMS-0653846.
} 


\begin{abstract}
We consider paths in the plane with $(1,0),(0,1)$, and $(a, b)$-steps that start at the origin, end at height $n$, and stay strictly to the left of a given non-decreasing right boundary. We show that if the boundary is periodic and has slope at most $b / a$, then the ordinary generating function for the number of such paths ending at height $n$ is algebraic. Our argument is in two parts. We use a simple combinatorial decomposition to obtain an Appell relation or "umbral" generating function, in which the power $z^{n}$ is replaced by a power series of the form $z^{n} \phi_{n}(z)$, where $\phi_{n}(0)=1$. Then we convert (in an explicit way) the umbral generating function to an ordinary generating function by solving a system of linear equations and a polynomial equation. This conversion implies that the ordinary generating function is algebraic.
\end{abstract}

\title{
1 Paths in the plane
}

A lattice path $\pi$ is a path in the plane with two kinds of steps: a unit north step $N$ or a unit east step $E$. Enumerating lattice paths and related combinatorial objects is closely related to calculating several probability distributions used in non-parametric statistics. See, for example, [12, 14]. If $x$ is a positive integer, a lattice path from the origin $(0,0)$ to the point $(x-1, n)$ can be coded by a length- $n$ non-decreasing sequence $\left(x_{0}, x_{1}, \ldots, x_{n-1}\right)$, where $0 \leq x_{i} \leq x-1$ and $x_{i}$ is the $x$-coordinate of the $(i+1)$-st north step. For example, the path EENENNEE is coded by $(2,3,3)$ and the horizontal path ending at $(x-1,0)$ is coded by the empty sequence.

Let $\underline{s}$ be a non-decreasing sequence with positive integer terms $s_{0}, s_{1}, s_{2}, \ldots$, thought of as a (right) boundary. A lattice path from $(0,0)$ to $(x-1, n)$ is an $\underline{s}$-lattice path if $x_{i}<s_{i}$ for $0 \leq i \leq n-1$. (Note that we require the path to stay strictly to the left of the boundary.) If $x \geq s_{n-1}$, then the number of $\underline{s}$-lattice paths from $(0,0)$ to $(x-1, n)$ does not depend on $x$. Let $\operatorname{LP}_{n}(\underline{s})$ be this common number, which equals the number of $\underline{s}$-lattice paths from $(0,0)$ to $\left(s_{n}-1, n\right)$. For instance, the sequence $\underline{i+1}$ with terms $1,2,3, \ldots$ gives a one-step staircase boundary. A $45^{\circ}$-rotation gives a bijection between $(\underline{i+1})$-lattice paths and Dyck paths. Thus, $\underline{s}$-lattice paths can be thought of as Dyck paths on a "bumpy" $x$-axis.

A boundary $\underline{s}$ is periodic of height $k$ and width $l$ if there is a finite non-decreasing sequence $\underline{s}_{0}$ with $k$ terms $s_{0}, s_{1}, \ldots, s_{k-1}$ such that $s_{k-1}=l$ and $s_{m}=s_{r}+q l$, where $q=$ $\lfloor m / k\rfloor$, and $r=m-q k$. Put another way, $\underline{s}$ is the concatenation $\underline{s}_{0}, \underline{s}_{0}+l, \underline{s}_{0}+2 l, \underline{s}_{0}+3 l, \ldots$. A boundary is ultimately periodic if it can be written as the concatenation $\underline{s}^{\prime}, p+\underline{s}$, where $\underline{s}^{\prime}$ is a finite initial sequence ending in $p$ and $\underline{s}$ is a periodic boundary.

In this paper, we present an elementary proof (avoiding the use of cathalitic variables and hence the kernel method) of a slightly more general form of a theorem of de Mier and 
Noy [11].

Theorem 1.1 Let $\underline{s}$ be an ultimately periodic right boundary. Then the ordinary generating function

$$
\sum_{n=0}^{\infty} \operatorname{LP}_{n}(\underline{s}) z^{n}
$$

is algebraic.

The proof begins with a combinatorial decomposition which first appeared in the enumeration of parking functions [6, 9]. This decomposition yields an Appell relation, an "umbral" generating function with the power $z^{n}$ replaced by a power series $z^{n} \phi_{n}(z)$. When $\underline{s}$ is ultimately periodic, the Appell relation can be converted to an ordinary generating function by solving a polynomial equation and a system of linear equations. The way this conversion is done implies that the ordinary generating function is algebraic. The method of obtaining generating functions from an Appell relation or functional equation is an old one and dates back, at least, to Pólya. Special cases of results in this paper can be find in [1, 2, 10, 17] and other papers.

Our method extends to paths allowing a third kind of steps. Let $a$ and $b$ be non-negative integers. An $(a, b)$-path $\pi$ is a path starting at the origin with three kinds of steps: $N, E$, and diagonal steps $D$ which go from a point $(u, v)$ to the point $(u+a, v+b)$. For example, the path EEDDNENNEE is a $(2,1)$-path starting at $(0,0)$ and ending at $(9,5)$. We allow $a$ and $b$ to be any non-negative integer. If $a=b=0$, then $D$ is a null step or "pause". If $a=0$ and $b=1$ or $a=1$ and $b=0$, then we have two kinds of $N$ or $E$ steps.

An $(a, b)$-path from $(0,0)$ to $(x-1, n)$ with $d$ diagonal steps can be coded by a pair of sequences. Let $\pi$ be such a path. Then there are $n-d(b-1)$ steps that are either $N$ or $D$. Replace every diagonal step $D$ by a $(0, b)$-step $C$ to obtain the compacted path. The compacted path goes from $(0,0)$ to $(x-d a-1, n)$. Record the $x$-coordinates where the $N$ or $C$ steps occur. This gives a non-decreasing sequence $\left(x_{0}, x_{1}, \ldots, x_{n-d(b-1)-1}\right)$ of length $n-d(b-1)$ with terms satisfying $0 \leq x_{i} \leq x-d a-1$. We also record the indices of the $C$ steps in this sequence. This is an increasing sequence $\left(y_{0}, y_{1}, \ldots, y_{d-1}\right)$ of length $d$ with terms satisfying $0 \leq y_{i} \leq n-d(b-1)-1$. For our example, the compacted path is EECCNENNEE and $((2,2,2,3,3),(0,1))$ codes the original path $\pi$.

We may also think of an $(a, b)$-path $\pi$ as a polygon or union of line segments. Inductively, if the path ends at the point $(u, v)$, an east step adds the unit horizontal line segment from $(u, v)$ to $(u+1, v)$, a north step adds the unit vertical line segment from $(u, v)$ to $(u, v+1)$, and a diagonal step adds the straight line segment from $(u, v)$ to $(u+a, v+b)$ (with slope $b / a)$. The right extent $\operatorname{Right}_{i}(\pi)$ of an $(a, b)$-path $\pi$ at $y$-coordinate $i$ is the $x$-coordinate of 
the rightmost point of the intersection of the path (as a polygon) with the horizontal line $y=i$. An $(a, b)$-path from $(0,0)$ to $(x-1, n)$ is a $(a, b ; \underline{s})$-path if for $0 \leq i \leq n-1$,

$$
\operatorname{Right}_{i}(\pi)<s_{i}
$$

As in the lattice path case, a $45^{\circ}$-rotation gives a bijection between $(1,1 ; \underline{i+1})$-paths and Schröder paths.

We begin this paper with a combinatorial decomposition for the set of all paths in a rectangle (Section 2). Using this decomposition, we derive recursions and Appell relations in Section 3. In Section 4, we show how to convert an Appell relation satisfying a periodic condition into an ordinary generating function. This method gives a proof of a generalization of Theorem 1.1 for $(a, b)$-paths. In Section 5, we work out a concrete example, giving another solution to the tennis ball problem. We derive explicit formulas for $(1, b)$-paths with an arithmetic-progression boundary in Section 6. Section 7 is devoted to analogs of our results for parking functions. We conclude, in Section 8, with some remarks and open problems.

This paper is self-contained for the most part. We shall only use the elementary and formal part of the theory of algebraic fractional power series. For background, see, for example, [16, Chapter 6].

\section{Combinatorial decomposition}

In this section, $\operatorname{Path}_{n}(\underline{s})$ denotes the set of all $(a, b ; \underline{s})$-paths or the set of $\underline{s}$-lattice paths, depending on the context, that end at $\left(s_{n}-1, n\right)$. If $y$ is a real number, let $\underline{y}$ be the constant sequence with terms $y, y, y, \ldots$.

Given a non-negative integer $a$ and a positive integer $b$, a boundary $\underline{s}$ satisfies the slope condition for $(a, b)$-paths if for every $j$,

$$
s_{j+1}>s_{j}-1+a / b, s_{j+2}>s_{j}-1+2 a / b, \ldots, s_{j+b}>s_{j}-1+a .
$$

We adopt the convention that the slope condition is satisfied for lattice paths and $(0,0)$ paths, and not satisfied for $(a, 0)$-paths if $a>0$. Thinking of the boundary as the union of the line segments from $\left(s_{i}, i\right)$ to $\left(s_{i+1}, i+1\right)$, the slope condition says that for every $j$, the line segment $\left(s_{j}-1, j\right)$ to $\left(s_{j}-1+a, j+b\right)$ (corresponding to a diagonal step) lies strictly above the right boundary. This implies that an $(a, b)$-path can only reach a point $\left(s_{i}, i\right)$ by an east step. This technical condition allows arguments for lattice paths to transfer without change to $(a, b)$-paths but is reasonably natural. (If the slope condition is not assumed, the situation is more complicated. However, there are analogs of our results for some cases.) 
For example, the boundary $i+1$ satisfies the slope condition for $(1,1)$-paths. More generally, when $\gamma \leq b / a$, the boundary $\lceil i / \gamma\rceil+1$ of integers immediately to the right of the line $y=\gamma(x-1)$, satisfies the slope condition for $(a, b)$-paths. See Figure 1 for two explicit examples.

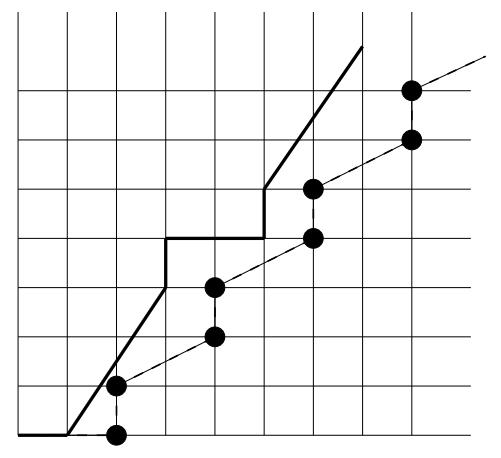

$$
\begin{aligned}
& a=2, b=3 \\
& s=2,2,4,4,6,6, \ldots
\end{aligned}
$$

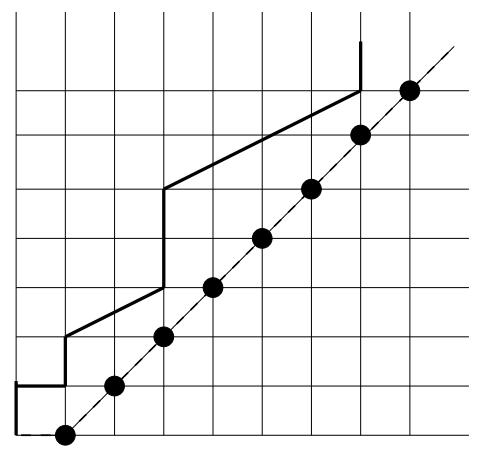

$$
\begin{aligned}
& a=2, b=1 \\
& s=1,2,3,4,5, \ldots
\end{aligned}
$$

Figure 1: Two examples of $(a, b ; \underline{s})$-paths. In the left-hand side diagram, the border satisfies the slope condition, whereas the border on the right-hand side does not.

Theorem 2.1 Let $\underline{s}$ be a right boundary satisfying the slope condition and $x$ be an integer such that $x>s_{n}$. Then there is a bijection:

$$
\operatorname{Path}_{n}(\underline{x}) \longleftrightarrow \bigcup_{m=0}^{n} \operatorname{Path}_{m}(\underline{s}) \times \operatorname{Path}_{n-m}\left(\underline{x-s_{m}}\right) .
$$

Proof. Observe that $\operatorname{Path}_{n}(\underline{x})$ is the set of all paths in the rectangle with lower left corner $(0,0)$ and upper right corner $(x-1, n)$. For a path $\pi$, let $m$ be the (unique) index

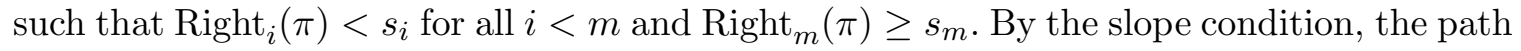
$\pi$ reaches the boundary by an east step. Thus, every path in $\operatorname{Path}_{n}(\underline{x})$ can be decomposed uniquely into two subpaths: a subpath in $\operatorname{Path}_{m}(\underline{s})$ and a subpath in the rectangle with corners $\left(s_{m}, m\right)$ and $(x-1, n)$. These paths are connected by an east step. To finish the proof, observe that paths in the rectangle with corners $\left(s_{m}, m\right)$ and $(x-1, n)$ are in bijection with paths in $\operatorname{Path}_{n-m}\left(\underline{x-s_{m}}\right)$. 


\section{Recursions and Appell relations}

The bijection of the previous section yields an infinite system of linear recursions, which are then written as an Appell relation. We first consider lattice paths. Since lattice paths are coded by non-decreasing sequences or multisets,

$$
\left|\operatorname{Path}_{n}(\underline{x})\right|=\left(\begin{array}{c}
x+n-1 \\
n
\end{array}\right)=(-1)^{n}\left(\begin{array}{c}
-x \\
n
\end{array}\right) .
$$

By Theorem 2.1, we have

$$
\sum_{m=0}^{n}(-1)^{m} \operatorname{LP}_{m}(\underline{s})\left(\begin{array}{c}
s_{m}-x \\
n-m
\end{array}\right)=\left(\begin{array}{c}
-x \\
n
\end{array}\right) .
$$

Since this recursion holds for all integers $x$ such that $x>s_{n}$, it holds as a polynomial identity in $x$. Setting $x=0$, we obtain, $\operatorname{LP}_{0}(\underline{s})=1$, and for $n \geq 1$,

$$
\sum_{m=0}^{n}(-1)^{m} \operatorname{LP}_{m}(\underline{s})\left(\begin{array}{c}
s_{m} \\
n-m
\end{array}\right)=0 .
$$

The next step is to multiply each of these equations by $t^{n}$ and sum over all $n$. Recall the following (easy) lemma, which can be found, for example, in [8].

Lemma 3.1 Suppose that the sequence $g_{n}$ is the solution to the triangular system

$$
\begin{aligned}
b_{0,0} g_{0} & =a_{0} \\
b_{1,1} g_{1}+b_{1,0} g_{0} & =a_{1} \\
b_{2,2} g_{2}+b_{2,1} g_{1}+b_{2,0} g_{0} & =a_{2} \\
& \vdots \\
b_{m, m} g_{m}+b_{m, m-1} g_{m-1}+b_{m, m-2} g_{m-2}+\cdots+b_{m, 1} g_{1}+b_{m, 0} g_{0} & =a_{m}
\end{aligned}
$$

where for all $m$, the diagonal coefficient $b_{m, m}$ is non-zero. Then

$$
\sum_{m=0}^{\infty} g_{m} t^{m} \phi_{m}(t)=\Psi(t)
$$

where

$$
\Psi(t)=\sum_{k=0}^{\infty} a_{k} t^{k} \text { and } \phi_{m}(t)=\sum_{k=0}^{\infty} b_{m+k, m} t^{k}
$$


Applying Lemma 3.1, we obtain

$$
1=\sum_{n=0}^{\infty}\left[\sum_{k=n}^{\infty}\left(\begin{array}{c}
s_{n} \\
k-n
\end{array}\right) t^{k}\right](-1)^{n} \operatorname{LP}_{n}(\underline{s})=\sum_{n=0}^{\infty}(-1)^{n} \operatorname{LP}_{n}(\underline{s}) t^{n}(1+t)^{s_{n}}
$$

Changing variables from $t$ to $-t$, we obtain an Appell relation for $\underline{s}$-lattice paths.

Lemma 3.2 For any boundary $\underline{s}$, the number of $\underline{s}$-lattice paths satisfy the equation

$$
\sum_{n=0}^{\infty} \operatorname{LP}_{n}(\underline{s}) t^{n}(1-t)^{s_{n}}=1
$$

This equation for $\underline{s}$-lattice paths has been obtained by Gessel [2]. Appell relations for $(a, b)$-paths are more complicated. We will enumerate $(a, b)$-paths weighted by the number of diagonal steps. Let

$$
\operatorname{SP}_{n}(a, b, \sigma ; \underline{s})=\sum_{d=0}^{\lfloor n / b\rfloor}\left|\operatorname{Path}_{n, d}(\underline{s})\right| \sigma^{d},
$$

where $\sigma$ is a variable and $\operatorname{Path}_{n, d}(\underline{s})$ is the set of $(a, b ; \underline{s})$-paths ending at $\left(s_{n}-1, n\right)$ and having $d$ diagonal steps. The (diagonal-step) enumerator is a polynomial in $\sigma$ except in the case $a=b=0$.

From the coding given in the introduction, the number of all $(a, b)$-paths with $d$ diagonal steps from $(0,0)$ to $(x-1, n)$ is

$$
(-1)^{n-d(b-1)}\left(\begin{array}{c}
-(x-d a) \\
n-d(b-1)
\end{array}\right)\left(\begin{array}{c}
n-d(b-1) \\
d
\end{array}\right) .
$$

Hence, the enumerator $\mathrm{SP}_{n}(a, b, \sigma ; \underline{x})$ of such paths is given by

$$
\operatorname{SP}_{n}(a, b, \sigma ; \underline{x})=\sum_{d=0}^{\lfloor n / b\rfloor}(-1)^{n-d(b-1)}\left(\begin{array}{c}
d a-x \\
n-d(b-1)
\end{array}\right)\left(\begin{array}{c}
n-d(b-1) \\
d
\end{array}\right) \sigma^{d} .
$$

Thus, by Theorem 2.1, for a right boundary $\underline{s}$ (satisfying the slope condition for $(a, b)$-paths),

$$
\sum_{m=0}^{n} \operatorname{SP}_{m}(a, b, \sigma ; \underline{s}) \mathrm{SP}_{n-m}\left(a, b, \sigma ; \underline{x-s_{m}}\right)=\operatorname{SP}_{n}(a, b, \sigma ; \underline{x}) .
$$


Setting $x=0$, we obtain the recursions (3.3):

$$
\begin{aligned}
& \sum_{m=0}^{n} \operatorname{SP}_{m}(a, b, \sigma ; \underline{s})\left(\sum_{j=0}^{\lfloor(n-m) / b\rfloor}(-1)^{n-m-j(b-1)}\left(\begin{array}{c}
s_{m}+j a \\
n-m-j(b-1)
\end{array}\right)\left(\begin{array}{c}
n-m-j(b-1) \\
j
\end{array}\right) \sigma^{j}\right) \\
= & \sum_{d=\lceil n /(a+b-1)\rceil}^{\lfloor n / b\rfloor}(-1)^{n-d(b-1)}\left(\begin{array}{c}
d a \\
n-d(b-1)
\end{array}\right)\left(\begin{array}{c}
n-d(b-1) \\
d
\end{array}\right) \sigma^{d} .
\end{aligned}
$$

To get the smaller range for the right sum, we use the fact that $\left(\begin{array}{l}c \\ d\end{array}\right) \neq 0$ if and only if $c \geq d$. We remark that determinantal formulas (similar to formulas given in [9]) for the enumerator of $(a, b)$-paths can be obtained with Cramer's rule from the recursions (3.3).

We use Lemma 3.1 again to express these recursions as an Appell relation. We start with the classic case of Schröder paths. When $a=b=1$, the sum on the right side of $\left(\mathrm{SR}_{n}\right)$ has one term, $(-1)^{n} \sigma^{n}$. Hence,

$$
\Psi(t)=\sum_{n=0}^{\infty}(-1)^{n} \sigma^{n} t^{n}=\frac{1}{1+\sigma t} .
$$

On the left side, the quantity $\operatorname{SP}_{m}(1,1, \sigma ; \underline{s})$ occurs multiplied by

$$
(-1)^{k}\left(\left(\begin{array}{c}
s_{m} \\
k
\end{array}\right)\left(\begin{array}{l}
k \\
0
\end{array}\right)+\left(\begin{array}{c}
s_{m}+1 \\
k
\end{array}\right)\left(\begin{array}{l}
k \\
1
\end{array}\right) \sigma+\left(\begin{array}{c}
s_{m}+2 \\
k
\end{array}\right)\left(\begin{array}{l}
k \\
2
\end{array}\right) \sigma^{2}+\ldots+\left(\begin{array}{c}
s_{m}+k \\
k
\end{array}\right)\left(\begin{array}{l}
k \\
k
\end{array}\right) \sigma^{k}\right),
$$

where $k=n-m$.

Using the easy binomial coefficient identity

$$
\left(\begin{array}{c}
s+i \\
k
\end{array}\right)\left(\begin{array}{c}
k \\
i
\end{array}\right)=\left(\begin{array}{c}
s+i \\
i
\end{array}\right)\left(\begin{array}{c}
s \\
k-i
\end{array}\right)
$$

we conclude that

$$
\begin{aligned}
\phi_{m}(t) & =(1-t)^{s_{m}}\left[1-\left(\begin{array}{c}
s_{m}+1 \\
1
\end{array}\right) \sigma t+\left(\begin{array}{c}
s_{m}+2 \\
2
\end{array}\right) \sigma^{2} t^{2}-\left(\begin{array}{c}
s_{m}+3 \\
3
\end{array}\right) \sigma^{3} t^{3}+\ldots\right] \\
& =(1-t)^{s_{m}}\left[\sum_{i=0}^{\infty}\left(\begin{array}{c}
-\left(s_{m}+1\right) \\
i
\end{array}\right) \sigma^{i} t^{i}\right] \\
& =(1-t)^{s_{m}}(1+\sigma t)^{-\left(s_{m}+1\right)} .
\end{aligned}
$$

This yields the Appell relation

$$
\sum_{n=0}^{\infty} \operatorname{SP}_{n}(1,1, \sigma ; \underline{s}) t^{n}\left(\frac{1-t}{1+\sigma t}\right)^{s_{n}}=1 .
$$


Setting $\sigma=0$, we recover the Appell relation for lattice paths in Lemma 3.2. Setting $\sigma=1$, we obtain an Appell relation for the total number of Schröder paths. Finally, setting $\sigma=-1$, we obtain

$$
\sum_{n=0}^{\infty} \mathrm{SP}_{n}(1,1,-1 ; \underline{s}) t^{n}=1 \text {. }
$$

This implies that when $n \geq 1, \operatorname{SP}_{n}(1,1,-1 ; \underline{s})=0$, that is, the number of Schröder paths from $(0,0)$ to $(n-1, n)$ with an even number of diagonal steps equals the number of such Schröder paths with an odd number of diagonal steps for any boundary $\underline{s}$ satisfying the slope condition for $(1,1)$-paths. For the case $s_{i}=i+1$, this is known. See [1, Prop. 2.1]. The two combinatorial proofs in [1] proving the special case can be applied without change to prove the general case.

By a similar argument, we obtain the following Appell relation for $(1, b)$-paths:

$$
\sum_{n=0}^{\infty} \operatorname{SP}_{n}(1, b, \sigma ; \underline{s}) t^{n}\left(\frac{1-t}{1+\sigma t^{b}}\right)^{s_{n}}=1
$$

For general $(a, b)$-paths, we have

$$
\phi_{m}(t)=(1-t)^{s_{m}}\left[\sum_{j=0}^{\infty}(-1)^{j}\left(\begin{array}{c}
s_{m}+a j \\
j
\end{array}\right)(1-t)^{j(a-1)} \sigma^{j} t^{b j}\right]
$$

and

$$
\Psi(t)=\sum_{d=0}^{\infty}(-1)^{d}\left(\begin{array}{c}
d a \\
d
\end{array}\right)(1-t)^{d(a-1)}\left(\sigma t^{b}\right)^{d} .
$$

The series $\phi_{m}(t)$ and $\Psi(t)$ can be simplified by the following lemma of Gould [3].

Lemma 3.3 For nonnegative integers $a$ and $s$,

$$
\sum_{j=0}^{\infty}\left(\begin{array}{c}
s+a j \\
j
\end{array}\right) z^{j}=\frac{f^{s+1}}{a+(1-a) f}
$$

where

$$
f(z)=\sum_{m=0}^{\infty} \frac{1}{a m+1}\left(\begin{array}{c}
a m+1 \\
m
\end{array}\right) z^{m}
$$

is the unique power series solution of the equation $f(z)-1=z[f(z)]^{a}$. 
There are at least two ways to prove Lemma 3.3. Gould's proof in [3] begins by proving

$$
\sum_{n=0}^{\infty}(-1)^{n}\left(\begin{array}{c}
s+a n \\
n
\end{array}\right) t^{n}(1+t)^{s+(a-1) n}=\frac{1}{1+a t}
$$

using the identities

$$
\sum_{i=0}^{n}(-1)^{i}\left(\begin{array}{c}
n \\
i
\end{array}\right)\left(\begin{array}{c}
s+a i \\
n
\end{array}\right)=(-1)^{n} a^{n} .
$$

These identities can be proved by a finite difference argument. The lemma then follows from the substitution $1+t=1 / f$ (so that $-t=(f-1) / f$ ), and $z=(f-1) / f^{a}$. Another way to prove Lemma 3.3 is to use the Lagrange inversion formula to show that the coefficient of $z^{j}$ in

$$
\frac{f^{s+1}}{a+(1-a) f}
$$

equals

$$
\frac{1}{n} \sum_{i=0}^{j-1}(a-1)^{j-i-1}\left(\begin{array}{c}
s+a j \\
i
\end{array}\right)(j a-i a+s),
$$

and then show by induction on $j$ that this sum equals $\left(\begin{array}{c}s+a j \\ j\end{array}\right)$.

Using Lemma 3.3, we obtain

$$
\phi_{n}(t)=\frac{F(t)}{a+(1-a) F(t)}((1-t) F(t))^{s_{n}}
$$

and

$$
\Psi(t)=\frac{F(t)}{a+(1-a) F(t)},
$$

where $F(t)=f\left(-(1-t)^{a-1} t^{b} \sigma\right)$.

Hence, for general $(a, b)$-paths we have the following Appell relation.

Lemma 3.4 Let $\underline{s}$ be a boundary satisfying the slope condition for $(a, b)$-paths. Then

$$
\sum_{n=0}^{\infty} \operatorname{SP}_{n}(a, b, \sigma ; \underline{s}) t^{n}[(1-t) F(t)]^{s_{n}}=1,
$$

where $F(t)=f\left(-(1-t)^{a-1} t^{b} \sigma\right)$ and $f(t)$ is the unique power series solution of $f(t)-1=$ $t f(t)^{a}$. 
In the next two sections, we show how to convert some Appell relations to ordinary generating functions. We motivate these results with the simplest case, when the boundary is an arithmetic progression. Let $s_{i}=c+i d$. For $(a, b)$-paths with $d \geq a / b$, the Appell relation can be written as

$$
\sum_{n=0}^{\infty} \operatorname{SP}_{n}(a, b, \sigma ; \underline{s}) z^{n}=\frac{1}{[(1-t) F(t)]^{c}},
$$

where $z=t[(1-t) F(t)]^{d}$. Since $t$ is an algebraic function of $z$ (see Lemma 4.1 below), we conclude that the ordinary generating function of $\operatorname{SP}_{n}(\sigma ; \underline{s})$ is algebraic. In the case of lattice paths, the Appell relation simplifies to

$$
\sum_{n=0}^{\infty} \operatorname{LP}_{n}(\underline{s}) z^{n}=\frac{1}{(1-t)^{c}},
$$

where $z=t(1-t)^{d}$. From this, we obtain the explicit formula

$$
\operatorname{LP}_{n}(\underline{c+i d})=\frac{c}{c+n(d+1)}\left(\begin{array}{c}
c+n(d+1) \\
n
\end{array}\right)
$$

This formula is known when $c=1$ (see, for example, [12, Section 1.5] or [16, p. 175]). In particular, the series $f(z)$ in Lemma 3.3 is the generating function $\sum_{m=0}^{\infty} \mathrm{LP}_{m}(\underline{1+a i}) z^{m}$. One way to derive this formula is to apply the Lagrange inversion formula (see, for example, [16, p. 42]) to equation (3.4). Another way is to use a difference operator theory, directly analogous to the differential operator theory for Gončarov polynomials developed in [9].

\section{Periodicity implies algebraicity}

In this section, we show how to obtain an ordinary generating function from an Appell relation when a periodicity condition is satisfied. We also show that this generating function is algebraic. We refer the reader to Section 6.1 of [16] for background on algebraic generating functions. Recall that a fractional power series is a series of the form

$$
\sum_{i=0}^{\infty} c_{i} z^{i / N}
$$

where $N$ is a fixed positive integer, the $c_{i}$ 's are complex numbers, and only non-negative powers of $z^{1 / N}$ occur. If a finite number of negative powers is allowed, we speak of fractional Laurent series. Puiseux's theorem asserts that the set of all fractional Laurent series is an algebraically closed field. We begin with a lemma asserting the existence of fractional power solutions of a given form for a functional equation. 
Lemma 4.1 Let $h(t)$ be a power series such that $h(0)=1$. Then the equation

$$
z=t^{k} h(t)
$$

has $k$ fractional power series solutions $\tau_{m}(z), 0 \leq m \leq k-1$ such that

$$
\tau_{0}(z)=z^{1 / k}+\sum_{i=2}^{\infty} c_{i} z^{i / k} \quad \text { and } \quad \tau_{m}(z)=\xi^{m} z^{1 / k}+\sum_{i=2}^{\infty} c_{i} \xi^{m i} z^{i / k}, 1 \leq m \leq k-1,
$$

where $\xi$ is a primitive $k$-th root of unity. Moreover, if $h(t)$ is algebraic, then $\tau_{0}(z), \ldots, \tau_{k-1}(z)$ are also algebraic.

Proof. The proof uses a standard argument. Suppose that $\tau(z)$ is a fractional series of the form $\sum_{i \geq 1} c_{i} z^{i / k}$; by equating coefficients on both sides of the equation $z=t^{k} h(t)$ we show that there are $k$ solutions of this form. Expand $\tau(z)^{k} h(\tau(z))$ as a fractional series. As $\tau(z)$ has no constant term,

$$
\tau(z)^{k} h(\tau(z))=c_{1}^{k} z+\sum_{j=1}^{\infty} f_{j}\left(c_{1}, c_{2}, \ldots, c_{j}, c_{j+1}\right) z^{1+j / k}
$$

where $f_{j}\left(c_{1}, c_{2}, \ldots, c_{j}, c_{j+1}\right)$ is a linear combination of terms of the form $c_{r_{1}} c_{r_{2}} \ldots c_{r_{l}}$ with $l \geq k, 1 \leq r_{1} \leq r_{2} \leq \ldots \leq r_{l}$, and $r_{1}+r_{2}+\cdots+r_{l}=j+k$. The only terms in $f_{j}\left(c_{1}, c_{2}, \ldots, c_{j}, c_{j+1}\right)$ containing $c_{j+1}$ is $c_{1}^{k-1} c_{j+1}$, with multiplicity $k$. Hence

$$
f_{j}\left(c_{1}, c_{2}, \ldots, c_{j}, c_{j+1}\right)=k c_{1}^{k-1} c_{j+1}+\tilde{f}_{j}\left(c_{1}, c_{2}, \ldots, c_{j}\right) .
$$

Equating coeffcients on both side of $z=\tau(z)^{k} h(\tau(z))$, we conclude that $c_{1}$ is a $k$-th root of unity. Once we have chosen the value of $c_{1}$, all the coefficients $c_{j+1}$ can be determined recursively by the equations $f_{j}\left(c_{1}, c_{2}, \ldots, c_{j+1}\right)=0$. Thus, there exist $k$ solutions, $\tau_{0}(z), \tau_{1}(z), \ldots, \tau_{k-1}(z)$, where the coefficient of $z^{1 / k}$ in $\tau_{m}(z)$ equals $\xi^{m}$. Suppose that

$$
\tau_{0}(z)=z^{1 / k}+\sum_{i=2}^{\infty} c_{i} z^{i / k}
$$

Then the coefficient of $z^{i / k}$ in $\tau_{m}(z)$ is $\xi^{m i} c_{i}$. This follows from the identity that

$$
f_{j}\left(c_{1} \xi^{m}, c_{2} \xi^{2 m}, \ldots, c_{j} \xi^{m j}, c_{j+1} \xi^{m(j+1)}\right)=\xi^{m(j+k)} f_{j}\left(c_{1}, c_{2}, \ldots, c_{j}, c_{j+1}\right) \xi^{m(j+k)} .
$$

To finish, suppose that $h(t)$ is algebraic. Then so is the series $t^{k} h(t)$. Let $Q(t, y)$ be a polynomial such that $Q\left(t, t^{k} h(t)\right)=0$. Then $Q\left(\tau_{m}(z), z\right)=0$ and hence, $\tau_{m}(z)$ is algebraic. 
If $h(t)$ is a polynomial, the equation $z=t^{k} h(t)$ may have fractional power series solutions with non-zero constant term, but if $h(t)$ has infinitely many terms, then $\tau_{0}(z), \tau_{1}(z), \ldots, \tau_{k-1}(z)$ are actually all the fractional power series solutions. Of course the equation may have solutions that are fractional Laurent series. Note also that the algebraic equation $P(z, t)$ appearing in the proof may have other fractional power series solutions besides $\tau_{0}(z), \ldots, \tau_{k-1}(z)$. These solutions satisfy $z=\tilde{g}(t)$, where $\tilde{g}(t)$ is a solution of $Q(t, y)=0$. For our purposes, we will only need existence of the $k$ solutions described in Lemma 4.1

Theorem 4.2 Let $b_{0}, b_{1}, \ldots, b_{k-1}$ be a sequence of non-negative integers of length $k, l=$ $b_{k-1}, s_{n}=q l+b_{j}$, where $n=q k+j$, and $\left(\ell_{n}\right)$ be a sequence satisfying the Appell relation

$$
\sum_{n=0}^{\infty} \ell_{n} t^{n} \phi(t)^{s_{n}}=\Psi(t),
$$

where $\phi(t)$ and $\Psi(t)$ are algebraic functions and $\phi(0)=1$. Then the ordinary generating function $\sum_{n=0}^{\infty} \ell_{n} z^{n}$ and the "section" generating functions $\sum_{q=0}^{\infty} \ell_{q k+j} z^{q}, 0 \leq j \leq k-1$, are algebraic.

Proof. Let $z=t^{k} \phi(t)^{l}$ and

$$
Q_{j}(z)=\sum_{q=0}^{\infty} \ell_{q k+j} z^{q} .
$$

Then we can rewrite the Appell relation in the following way:

$$
\begin{aligned}
\Psi(t) & =\sum_{j=0}^{k-1} \sum_{q=0}^{\infty} \ell_{q k+j} t^{q k+j} \phi(t)^{q l+b_{j}} \\
& =\sum_{j=0}^{k-1} Q_{j}(z) t^{j} \phi(t)^{b_{j}} .
\end{aligned}
$$

By Lemma 4.1, there are $k$ solutions $\tau_{i}(z)$ of the form $\xi^{i} z^{1 / k}+\sum_{n=2}^{\infty} c_{n} z^{n / k}$, where $\xi$ is a primitive $k$-th root of unity. Substituting the solutions $\tau_{i}(z)$ into the Appell relation, we get $k$ linear equations for $Q_{j}(z)$ :

$$
\left(\begin{array}{ccccc}
\phi\left(\tau_{0}\right)^{b_{0}} & \tau_{0} \phi\left(\tau_{0}\right)^{b_{1}} & \tau_{0}^{2} \phi\left(\tau_{0}\right)^{b_{2}} & \ldots & \tau_{0}^{k-1} \phi\left(\tau_{0}\right)^{b_{k-1}} \\
\phi\left(\tau_{1}\right)^{b_{0}} & \tau_{1} \phi\left(\tau_{1}\right)^{b_{1}} & \tau_{1}^{2} \phi\left(\tau_{1}\right)^{b_{2}} & \ldots & \tau_{1}^{k-1} \phi\left(\tau_{1}\right)^{b_{k-1}} \\
\vdots & \vdots & \vdots & \ddots & \vdots \\
\phi\left(\tau_{k-1}\right)^{b_{0}} & \tau_{k-1} \phi\left(\tau_{k-1}\right)^{b_{1}} & \tau_{k-1}^{2} \phi\left(\tau_{k-1}\right)^{b_{2}} & \cdots & \tau_{k-1}^{k-1} \phi\left(\tau_{k-1}\right)^{b_{k-1}}
\end{array}\right)\left(\begin{array}{c}
Q_{0}(z) \\
Q_{1}(z) \\
\vdots \\
Q_{k-1}(z)
\end{array}\right)=\left(\begin{array}{c}
\Psi\left(\tau_{0}\right) \\
\Psi\left(\tau_{1}\right) \\
\vdots \\
\Psi\left(\tau_{k-1}\right)
\end{array}\right)
$$


The $k \times k$ matrix on the left is non-singular. Indeed, the lowest power of $z$ occurring in its determinant is $z^{(k-1) / 2}$ and that power has coefficient equal to the Vandermonde determinant $\operatorname{det}\left[\xi^{i j}\right]_{0 \leq i, j \leq k-1}$. Thus, we can solve the system of linear equations and obtain $Q_{j}(z)$ as rational functions in $\tau_{0}(z), \tau_{1}(z), \ldots, \tau_{k-1}(z)$. Since the series $\tau_{i}(z)$ are algebraic, the functions $Q_{j}(z)$ and the sum $Q_{0}\left(z^{k}\right)+z Q_{1}\left(z^{k}\right)+\cdots+z^{k-1} Q_{k-1}\left(z^{k}\right)$, which equals $\sum_{n=0}^{\infty} l_{n} z^{n}$, are also algebraic.

The algebraicity of the generating function for $(a, b)$-paths with an ultimately periodic boundary follows from the previous theorem.

Corollary 4.3 Let $\underline{s}$ be a ultimately periodic right boundary having height $k$ satisfying the slope condition for $(a, b)$-paths. Then the ordinary generating function

$$
\sum_{n=0}^{\infty} \mathrm{SP}_{n}(a, b, \sigma ; \underline{s}) z^{n}
$$

is algebraic. In addition, if the initial segment has length $r$, then the section generating functions

$$
\sum_{q=r}^{\infty} \operatorname{SP}_{q k+j+r}(a, b, \sigma ; \underline{s}) z^{q}, \quad j=0,1,2, \ldots, k-1
$$

are algebraic.

Proof. Suppose that the boundary $\underline{s}$ is the concatenation $\underline{a}, a_{r-1}+\underline{s^{\prime}}$, where $\underline{a}$ is the initial segment $a_{0}, \ldots, a_{r-1}$, and $\underline{s}^{\prime}$ is a periodic boundary. Then the Appell relation for $\underline{s}$ is

$\sum_{i=0}^{r-1} \mathrm{SP}_{i}(a, b, \sigma ; \underline{a}) t^{i}[(1-t) F(t)]^{a_{i}}+t^{r}[(1-t) F(t)]^{a_{r-1}} \sum_{n=0}^{\infty} \mathrm{SP}_{n+r}(a, b, \sigma ; \underline{s}) t^{n}[(1-t) F(t)]^{s_{n}^{\prime}}=1$,

where $F(t)$ is the series defined in Lemma 3.4. By Theorem 4.2, the partial generating function $\sum_{n=0}^{\infty} \mathrm{SP}_{n+r}(a, b, \sigma ; \underline{s}) z^{n}$ as well as the analogous section generating functions are algebraic. Since the full generating function $\sum_{n=0}^{\infty} \mathrm{SP}_{n}(a, b, \sigma ; \underline{s})$ can be obtained by multiplying the partial generating function by $z^{r}$ and adding the polynomial $\sum_{i=0}^{r-1} \mathrm{SP}_{i}(a, b, \sigma ; \underline{a}) z^{i}$, the full generating function is algebraic.

Corollary 4.3 includes the case of lattice paths. Just set $\sigma=0$. Thus, we have proved Theorem 1.1.

As observe earlier, when $\gamma$ is a rational number, the boundary $\lceil n / \gamma\rceil+1$ is periodic and hence, the generating functions $\sum_{n=0}^{\infty} \operatorname{LP}_{n}(\lceil n / \gamma\rceil+1) z^{n}$ is algebraic. What can be said about the generating functions $\sum_{n=0}^{\infty} \operatorname{LP}_{n}\left(\underline{(\overline{\lceil n / \gamma\rceil+1})} z^{n}\right.$ when $\gamma$ is an algebraic or transcendental number? 


\section{The tennis ball problem revisited}

The proof of Theorem 4.2 provides a recipe for calculating explicitly the ordinary generating functions. We show how this might be done with a concrete example. Let $\underline{s}$ be the ultimately periodic boundary

$$
1, \overbrace{l+1, \ldots, l+1}^{k \text { times }}, \overbrace{2 l+1, \ldots, 2 l+1}^{k \text { times }}, \overbrace{3 l+1, \ldots, 3 l+1}^{k \text { times }}, \ldots
$$

of height $k$ and width $l$. The generating function $Q_{0}(z)=\sum_{q=0}^{\infty} \operatorname{LP}_{q k+1}(\underline{s}) z^{q}$ for this boundary and ordinary lattice paths yields a solution to the generalized tennis ball problem (posed in [4, 10] and solved in [11]). We note that there are other choices of boundary, such as

$$
\overbrace{l+1, \ldots, l+1}^{k \text { times }}, \overbrace{2 l+1, \ldots, 2 l+1}^{k \text { times }}, \overbrace{3 l+1, \ldots, 3 l+1}^{k \text { times }}, \ldots
$$

that would solve the problem.

Separating the length-1 initial segment from the periodic part, we have the Appell relation

$$
(1-t)+\sum_{j=0}^{k-1} Q_{j}(z) t^{j+1}(1-t)^{l+1}=1,
$$

where $z=t^{k}(1-t)^{l}$ and $Q_{j}(z)=\sum_{q=0}^{\infty} \operatorname{LP}_{1+q k+j}(\underline{s}) z^{q}$. Let $\tau_{m}$, for $0 \leq m \leq k-1$, be the $k$ solutions of the equation $z=t^{k}(1-t)^{l}$ that are fractional power series with zero constant term. Using the relation $\left(1-\tau_{m}\right)^{l}=z / \tau_{m}^{k}$, we obtain the system of linear equations

$$
\left(\begin{array}{ccccc}
z\left(1-\tau_{0}\right) \tau_{0}^{1-k} & z\left(1-\tau_{0}\right) \tau_{0}^{2-k} & z\left(1-\tau_{0}\right) \tau_{0}^{3-k} & \cdots & z\left(1-\tau_{0}\right) \\
z\left(1-\tau_{1}\right) \tau_{1}^{1-k} & z\left(1-\tau_{1}\right) \tau_{1}^{2-k} & z\left(1-\tau_{1}\right) \tau_{1}^{3-k} & \cdots & z\left(1-\tau_{1}\right) \\
\vdots & \vdots & \vdots & \ddots & \vdots \\
z\left(1-\tau_{k-1}\right) \tau_{k-1}^{1-k} & z\left(1-\tau_{k-1}\right) \tau_{k-1}^{2-k} & z\left(1-\tau_{k-1}\right) \tau_{k-1}^{3-k} & \cdots & z\left(1-\tau_{k-1}\right)
\end{array}\right)\left(\begin{array}{c}
Q_{0}(z) \\
Q_{1}(z) \\
\vdots \\
Q_{k-1}(z)
\end{array}\right)=\left(\begin{array}{c}
\tau_{0} \\
\tau_{1} \\
\vdots \\
\tau_{k-1}
\end{array}\right)
$$

We will now solve the system using Cramer's rule. To do this, we need to compute two determinants. First, the determinant of the $k \times k$ matrix on the left equals

$$
z^{k} \prod_{j=0}^{k-1} \tau_{j}^{1-k}\left(1-\tau_{j}\right) \operatorname{det}\left(\begin{array}{ccccc}
1 & \tau_{0} & \tau_{0}^{2} & \cdots & \tau_{0}^{k-1} \\
1 & \tau_{1} & \tau_{1}^{2} & \cdots & \tau_{1}^{k-1} \\
\vdots & \vdots & \vdots & \ddots & \vdots \\
1 & \tau_{k-1} & \tau_{k-1}^{2} & \cdots & \tau_{k-1}^{k-1}
\end{array}\right)
$$


Hence, by the formula for the Vandermonde determinant, this determinant equals

$$
z^{k} \prod_{j=0}^{k-1} \tau_{j}^{1-k}\left(1-\tau_{j}\right) \prod_{0 \leq i<j \leq k-1}\left(\tau_{j}-\tau_{i}\right)
$$

To compute, say, $Q_{0}(z)$, also need the determinant

$$
\operatorname{det}\left(\begin{array}{ccccc}
\tau_{0} & z\left(1-\tau_{0}\right) \tau_{0}^{2-k} & z\left(1-\tau_{0}\right) \tau_{0}^{3-k} & \cdots & z\left(1-\tau_{0}\right) \\
\tau_{1} & z\left(1-\tau_{1}\right) \tau_{1}^{2-k} & z\left(1-\tau_{1}\right) \tau_{1}^{3-k} & \cdots & z\left(1-\tau_{1}\right) \\
\vdots & \vdots & \vdots & \ddots & \vdots \\
\tau_{k-1} & z\left(1-\tau_{k-1}\right) \tau_{k-1}^{2-k} & z\left(1-\tau_{k-1}\right) \tau_{k-1}^{3-k} & \cdots & z\left(1-\tau_{k-1}\right)
\end{array}\right) .
$$

This determinant equals

$$
z^{k-1} \prod_{j=0}^{k-1} \tau_{j}^{2-k}\left(1-\tau_{j}\right) \operatorname{det}\left(\begin{array}{ccccc}
\frac{\tau_{0}^{k-1}}{1-\tau_{0}} & 1 & \tau_{0} & \cdots & \tau_{0}^{k-2} \\
\frac{\tau_{1}^{k-1}}{1-\tau_{1}} & 1 & \tau_{1} & \cdots & \tau_{1}^{k-2} \\
\vdots & \vdots & \vdots & \ddots & \vdots \\
\frac{\tau_{k-1}^{k-1}}{1-\tau_{k-1}} & 1 & \tau_{k-1} & \cdots & \tau_{k-1}^{k-2}
\end{array}\right)
$$

\section{Lemma 5.1}

$$
\operatorname{det}\left(\begin{array}{ccccc}
\frac{\tau_{0}^{k-1}}{1-\tau_{0}} & 1 & \tau_{0} & \cdots & \tau_{0}^{k-2} \\
\frac{\tau_{1}^{k-1}}{1-\tau_{1}} & 1 & \tau_{1} & \cdots & \tau_{1}^{k-2} \\
\vdots & \vdots & \vdots & \ddots & \vdots \\
\frac{\tau_{k-1}^{k-1}}{1-\tau_{k-1}} & 1 & \tau_{k-1} & \cdots & \tau_{k-1}^{k-2}
\end{array}\right)=(-1)^{k+1} \frac{\prod_{0 \leq i<j \leq k-1}\left(\tau_{j}-\tau_{i}\right)}{\prod_{j=0}^{k-1}\left(1-\tau_{j}\right)}
$$

The evaluation of the determinant can be done in many ways, for example, as a special case of Theorem (Cauchy+) in [5] or Theorem 25 in [7], or by the theory of alternants and symmetric functions, as described in [13, Chap. 11]. We include a brief proof here for the convenience of the reader.

Proof. Expanding the determinant along the first column, we conclude that it equals the sum

$$
\sum_{j=0}^{k-1}(-1)^{j+1} \frac{\tau_{j}^{k-1}}{1-\tau_{j}} \prod_{\substack{0 \leq i<i^{\prime} \leq k-1 \\ i, i^{\prime} \neq j}}\left(\tau_{i^{\prime}}-\tau_{i}\right)
$$


We will obtain this sum in a different way. From the following expansion of a singular matrix along the first column,

$$
\begin{aligned}
0 & =\operatorname{det}\left(\begin{array}{ccccc}
\tau_{0}^{k-1} & 1 & \tau_{0} & \ldots & \tau_{0}^{k-1} \\
\tau_{1}^{k-1} & 1 & \tau_{1} & \ldots & \tau_{1}^{k-1} \\
\vdots & \vdots & \vdots & \ddots & \vdots \\
\tau_{k-1}^{k-1} & 1 & \tau_{k-1} & \ldots & \tau_{k-1}^{k-1} \\
1 & 1 & 1 & \ldots & 1
\end{array}\right) \\
& =\left(\sum_{j=0}^{k-1}(-1)^{j} \tau_{j}^{k-1} \prod_{\substack{0 \leq i<i^{\prime} \leq k-1 \\
i, i^{\prime} \neq j}}\left(\tau_{i^{\prime}}-\tau_{i}\right) \prod_{i \neq j}\left(1-\tau_{i}\right)\right)+(-1)^{k} \prod_{0 \leq i<j \leq k-1}\left(\tau_{j}-\tau_{i}\right),
\end{aligned}
$$

we conclude, on dividing both sides by $\prod_{j=0}^{k-1}\left(1-\tau_{j}\right)$, that

$$
\sum_{j=0}^{k-1}(-1)^{j+1} \frac{\tau_{j}^{k-1}}{1-\tau_{j}} \prod_{\substack{0 \leq i<i^{\prime} \leq k-1 \\ i, i^{\prime} \neq j}}\left(\tau_{i^{\prime}}-\tau_{i}\right)=(-1)^{k+1} \frac{\prod_{0 \leq i<j \leq k-1}\left(\tau_{j}-\tau_{i}\right)}{\prod_{j=0}^{k-1}\left(1-\tau_{j}\right)}
$$

Using Cramer's rule and Lemma 5.1, we obtain, after some cancellations,

$$
Q_{0}(z)=\frac{(-1)^{k+1}}{z} \prod_{j=0}^{k-1} \frac{\tau_{j}(z)}{1-\tau_{j}(z)}
$$

Thus, we have the following theorem, derived earlier in [11].

Theorem 5.2 Let $\underline{s}$ be the ultimately periodic boundary in the tennis ball poblem. Then

$$
\sum_{q=0}^{\infty} \operatorname{LP}_{k q+1}(\underline{s}) z^{q}=\frac{(-1)^{k+1}}{z} \prod_{j=0}^{k-1} \frac{\tau_{j}(z)}{1-\tau_{j}(z)},
$$

where $\tau_{0}(z), \tau_{1}(z), \ldots, \tau_{k-1}(z)$ are the $k$ fractional power series solutions in $z^{1 / k}$ with zero constant term to the equation $z=t^{k}(1-t)^{l}$.

Our formula appears to be different from the one given in [11, Theorem 1]. The formula in that paper is

$$
Q_{0}(z)=\frac{-1}{z} \prod_{j=1}^{k}\left(1-w_{j}(z)\right)
$$


where $w_{1}(z), w_{2}(z), \ldots, w_{k}(z)$ are the $k$ fractional power series solutions of the equation $(w-1)^{k}-z w^{k+l}=0$. We can reorder the solutions so that $w_{j-1}=1 /\left(1-\tau_{j}\right)$. Thus, the two formulas are equivalent.

As was done in [11, an explicit formula can be obtained when $k=l$. The solution to the equation $y=t(1-t)$ that does not have constant term is the power series $H(y)$, where

$$
H(y)=\frac{1}{2}(1-\sqrt{1-4 y})=y-2 y^{2}+\ldots .
$$

Observing that the equation $z=t^{k}(1-t)^{k}$ can be formally rewritten as to $z^{1 / k}=t(1-t)$, we conclude that $H\left(z^{1 / k}\right), H\left(\xi z^{1 / k}\right), H\left(\xi^{2} z^{1 / k}\right), \ldots, H\left(\xi^{k-1} z^{1 / k}\right)$, are $k$ fractional power series solutions to $z=t^{k}(1-t)^{k}$ with zero constant term. Since

$$
\frac{H(y)}{1-H(y)}=\frac{1-\sqrt{1-4 y}}{1+\sqrt{1-4 y}}=\frac{1-\sqrt{1-4 y}}{2 y}-1
$$

we obtain the the following theorem.

Corollary 5.3 Let $\underline{s}$ be the tennis ball boundary with $k=l$. Then

$$
\sum_{q=0}^{\infty} \operatorname{LP}_{k q}(\underline{s}) z^{q}=\frac{(-1)^{k+1}}{z} \prod_{j=0}^{k-1}\left(C\left(\xi^{j} z^{1 / k}\right)-1\right)
$$

where

$$
C(y)=\frac{1-\sqrt{1-4 y}}{2 y},
$$

the generating function for the Catalan numbers, and $\xi=e^{2 \pi i / k}$.

When $k=l=2$, Corollary 5.3 specializes (after simple manipulations) to formulas given earlier in [10] and [11]. The simple form of the generating function suggests that after all, there may be an elementary combinatorial solution to the tennis ball problem, at least in the case $k=l$.

We can also find formulas for $Q_{m}(z)$ when $m \geq 1$. These generating functions involve more complicated symmetric functions in $\tau_{0}(z), \tau_{1}(z), \ldots, \tau_{k-1}(z)$. For example, when $k=$ $l=2$,

$$
\begin{aligned}
Q_{1}(z) & =\frac{4}{z(1+\sqrt{1+4 z})(1+\sqrt{1-4 z})}-1 \\
& =3+22 z+211 z^{2}+2306 z^{3}+23270 z^{4}+338444 z^{5}+\cdots
\end{aligned}
$$


We remark that the boundaries in the tennis ball problem do not satisfy the slope condition for $(a, b)$-paths when $a \geq 1$. There are complicated solutions to the analogs of the tennis ball problem for for $(1, b)$-paths.

\section{Explicit formulas for $(1, b)$-paths}

As remarked earlier, $(1,1)$-paths with the boundary $1,2,3, \ldots$ are in bijection with Schrörder paths, which have been studied in [1] and [12]. In this section, we derive explicit formulas for $(1, b)$-paths with the arithmetic-progression boundary $c, c+d, c+2 d, \ldots$, where $c$ and $d$ are positive integers.

We use the Appell relation

$$
\sum_{n=0}^{\infty} \mathrm{SP}_{n}(1, b, \sigma ; \underline{c+i d})\left[t\left(\frac{1-t}{1+\sigma t^{b}}\right)^{d}\right]^{n}=\left(\frac{1+\sigma t^{b}}{1-t}\right)^{c}
$$

derived in Section 3. When $b=d=1$, then we can solve the quadratic equation $z(1+\sigma t)=$ $t(1-t)$ explicitly. Choosing the right solution and observing that $(1+\sigma t) /(1-t)=t / z$, we obtain

$$
\sum_{n=0}^{\infty} \mathrm{SP}_{n}(1,1, \sigma ; \underline{c+i}) z^{n}=\left[\frac{1-\sigma z-\sqrt{(1-\sigma z)^{2}-4 z^{2}}}{2 z}\right]^{c} .
$$

When $c=1$, this formula is given in [1, p. 44]. We note that the general case (with arbitrary $c$ ) is derivable by an easy combinatorial argument from the case $c=1$. In principle, one can also obtain explicit formulas when $d=2$ or 3 using formulas for solving cubic and quartic equations.

For general $b, c$, and $d$, we can obtain explicit formulas for the diagonal-step enumerator using the Lagrange inversion formula. This formula says that

$$
n \mathrm{SP}_{n}(1, b, \sigma ; \underline{c+i d})=\left[t^{n-1}\right] \frac{d}{d t}\left[\left(\frac{1+\sigma t^{b}}{1-t}\right)^{c}\right]\left(\frac{1+\sigma t^{b}}{1-t}\right)^{n d},
$$

where $\left[t^{n-1}\right]$ abbreviates "the coefficient of $t^{n-1}$ in the series". The series on the right side is

$$
c\left(1+b \sigma t^{b-1}+(\sigma-b \sigma) t^{b}\right) \frac{\left(1+\sigma t^{b}\right)^{c+n d-1}}{(1-t)^{c+n d+1}} .
$$

Using the binomial theorem, we obtain

$$
\mathrm{SP}_{n}(1, b, \sigma ; \underline{c+i d})=\frac{c}{n}\left[T_{n, n-1}(\sigma)+\sigma b T_{n, n-b+1}(\sigma)+\sigma(1-b) T_{n, n-b}(\sigma)\right],
$$


where $T_{n, m}(\sigma)$ is the polynomial (with parameters $b, c, d$ ) defined by

$$
\sum_{m=0}^{\infty} T_{n, m}(\sigma) t^{m}=\frac{\left(1+\sigma t^{b}\right)^{c+n d-1}}{(1-t)^{c+n d+1}} .
$$

Explicitly,

$$
T_{n, m}(\sigma)=\sum_{j=0}^{\lfloor m / b\rfloor}\left(\begin{array}{c}
c+n d-1 \\
j
\end{array}\right)\left(\begin{array}{c}
c+n d+m-b j \\
m-b j
\end{array}\right) \sigma^{j} .
$$

In particular, when $b=1$, we obtain

$$
\mathrm{SP}_{n}(1,1, \sigma ; \underline{c+i d})=\frac{c(\sigma+1)}{n} \sum_{j=0}^{n-1}\left(\begin{array}{c}
c+n d-1 \\
j
\end{array}\right)\left(\begin{array}{c}
c+(n+1) d-j-1 \\
(n-1)-j
\end{array}\right) \sigma^{j} .
$$

\section{$7 \quad$ Parking functions}

Parking functions are rearrangements of lattice paths. An s-parking function of length $n$ is a sequence $\left(x_{0}, x_{1}, \ldots, x_{n-1}\right)$ of non-negative integers such that its rearrangement $\left(x_{(0)}, x_{(1)}, \ldots, x_{(n-1)}\right)$ into a non-decreasing sequence satisfies the inequalities: $x_{(i)}<s_{i}$, that is, the non-decreasing rearrangement is an $\underline{s}$-lattice path. In this section, we briefly describe analogs of our results for parking functions. We shall freely use results from [9].

Let $\mathrm{P}_{n}(\underline{s})$ be the number of $\underline{s}$-parking functions of length $n$. Then the following Appell relation holds [9, Section 3]:

$$
1=\sum_{n=0}^{\infty} \mathrm{P}_{n}(\underline{s}) \frac{t^{n} e^{-s_{n} t}}{n !}
$$

Theorem 7.1 Let $\underline{s}$ be a ultimately periodic right boundary. Then the exponential generating function

$$
\sum_{n=0}^{\infty} \mathrm{P}_{n}(\underline{s}) z^{n} / n !
$$

is algebraic over the extension field $\mathbb{C}(z)\langle T\rangle$, obtained by adding to the field of complex rational functions $\mathbb{C}(z)$ the set $T=\left\{\tau_{0}(z), \tau_{1}(z), \ldots, \tau_{k-1}(z)\right\}$ of the $k$ solutions to the equation $z=t^{k} e^{-l t}$ that are fractional power series in $z^{1 / k}$.

Proof. The argument in the proof of Theorem 4.2 can be recycled. The only thing we need to check that is that $y_{m}=e^{-\tau_{m}(z)}$ is algebraic over $\mathbb{C}(z)\langle T\rangle$, but this follows since $y_{m}^{l}=z / \tau_{m}^{k}$. 


\section{Paths not taken}

The methods in this paper can be applied to other kinds of paths (and objects related to paths). For example, one can consider $(a / k, b / k)$-paths with fractional diagonal steps, that is, paths with $E, N$, and $(a / k, b / k)$ steps, where $a$ and $b$ are non-negative integers and $k$ is a positive integer such that the greatest common divisor of $a, b, k$ is 1 . When $a=b=1$ and $k=2$, then $(a / k, b / k)$-paths to $(n, n)$ with boundary $1,2,3, \ldots$ are in bijection with Motzkin paths from $(0,0)$ to $(2 n, 2 n)$ as defined in [16, Ex. 6.38c, p. 238]. The theory extends naturally to such paths.

It is easy to find boundaries for which the generating function $\sum \mathrm{LP}_{n}(\underline{s}) z^{n}$ is nonalgebraic. By [16, Chapter 6], an algebraic power seris is D-finite and the coefficients of a D-finite power series satisfy a polynomial recursion. As $\operatorname{LP}_{n}(\underline{s})$ is an increasing sequence of positive integers, there is a polynomial $P(n)$ such that

$$
\mathrm{LP}_{n}(\underline{s}) \leq P(n) P(n-1) \cdots P(2) P(1)
$$

if $\sum \mathrm{LP}_{n}(\underline{s}) z^{n}$ is D-finite. Thus, if $\underline{s}$ is a sequence which increases sufficiently rapidly, then the generating function $\sum \mathrm{LP}_{n}(\underline{s}) z^{n}$ is not D-finite, and hence, not algebraic. However, the finer details are elusive. For example, we do not have an explicit example of a boundary with a non-algebraic but D-finite generating function, although it is reasonable to conjecture that one exists. It is also unknown whether there are boundaries $\underline{s}$ which are not ultimately periodic with an algebraic generating function $\sum \mathrm{LP}_{n}(\underline{s}) z^{n}$. To conclude with a concrete problem, is the generating function for $\operatorname{LP}_{n}(1,2,2,3,3,3,4,4,4,4, \ldots)$, where there are one 1 , two 2's, three 3's, and, in general, $n$ n's in the boundary, algebraic or D-finite?

Acknowledgement. We thank Marc Noy for many insightful discussions. Lemma 3.4 was found by trial and error with the help of MAPLE, the package EKHAD in [15], and the On-line encyclopedia of integer sequences. We thank Christian Krattenthaler for directing us to the paper [3] of Gould, where the lemma was derived earlier.

\section{References}

1. J. Bonin, L. Shapiro, R. Simion, Some $q$-analogues of the Schröder numbers arising from combinatorial statistics on lattice paths, J. Statist. Plann. Inference, 34 (1993) 35-55. 
2. I. Gessel, A probabilistic method for lattice path enumeration, J. Statist. Plann. Inference, 14 (1986) 49-58.

3. H.W. Gould, Some generalizations of Vandermonde's convolution, Amer. Math. Monthly, 63 (1956) 84-91.

4. R.P. Grimaldi, J.G. Moser, The Catalan numbers and a tennis ball problem, Congr. Numer. 125 (1997) 65-71.

5. G.N. Han, C. Krattenthaler, Rectangular Scott-type permanents, Sém. Lothar. Combin. 43 (1999), Art. B43g.

6. A.G. Konheim, B. Weiss, An occupancy discipline and applications, SIAM J. Appl. Math. 14(1966) 1266-1274.

7. C. Krattenthaler, Advanced determinant calculus: a complement, Linear Algebra Appl. 411 (2005), 68-166.

8. J.P.S. Kung, X. Sun, C. Yan, Two-boundary lattice paths and parking functions, Adv. Appl. Math., to appear.

9. J.P.S. Kung, C. Yan, Gončarov polynomials and parking functions, J. Combin. Theory Ser. A, 102(2003), 16-37.

10. D. Merlini, R. Sprugnoli, M.C. Verri, The tennis ball problem, J. Combin. Theory Ser. A 99 (2002) 307-344.

11. A. de Mier, M. Noy, A solution to the tennis ball problem, Theoret. Comput. Sci. 346 (2005) 254-264.

12. S.G. Mohanty, Lattice Path Counting and Applications, Academic Press, New York, 1979 .

13. T. Muir, W.H. Metzler, A treatise on the theory of determinants, revised edition, Longmans, London, 1933; reprinted, Dover, New York, 1960.

14. H. Niederhausen, Sheffer polynomials for computing exact Kolmogorov-Smirnov and Rényi type distributions, Ann. Statist. 9 (1981), 923-944.

15. M. Petkovšek, H. S. Wilf, D. Zeilberger, $A=B$, A K Peters, Wellesley MA, 1996.

16. R.P. Stanley, Enumerative Combinatorics, Volume 2. Cambridge University Press, Cambridge, 1999.

17. U. Tamm, Lattice paths not touching a given boundary, J. Statist. Plann. Inference, 105 (2002) 433-448. 